\title{
Improving our family tree
}

Our understanding of human evolution would be improved substantially by having a well-resolved phylogenetic tree of primates - a tree that, because of its complexity, has been difficult to trace. Now, an international team of molecular taxonomists has cracked the problem by sequencing a large, multigene data set from representatives of $90 \%$ of primate genera. The resulting phylogeny is robust, clears up long-standing controversies and sheds new light on human evolution.

Molecular-genetic studies of primate phylogeny have generally relied on sequences from single genes or from uniparentally inherited mitochondrial DNA that are taken from small numbers of primate taxa. These molecular data are then combined with other data such as biogeography and morphology to infer phylogeny. The pattern of speciation that can be inferred from such limited data is restricted to local phylogenies, whereas broader radiations are riddled with ambiguities because of confounding factors such as rapid speciation, differing rates of genome divergence between lineages, and hybridization between species inhabiting the same geographic region. The new study vastly expands our limited molecular data set by amplifying a genome-wide set of 54 nuclear-gene regions (across Y chromosomes, $\mathrm{X}$ chromosomes and autosomes) in 186 primate and five outgroup species, totalling $\sim 8 \mathrm{Mb}$ of sequence.

The tree constructed from these data is robust (90-100\% bootstrap support) and provides valuable information on both recent and deep

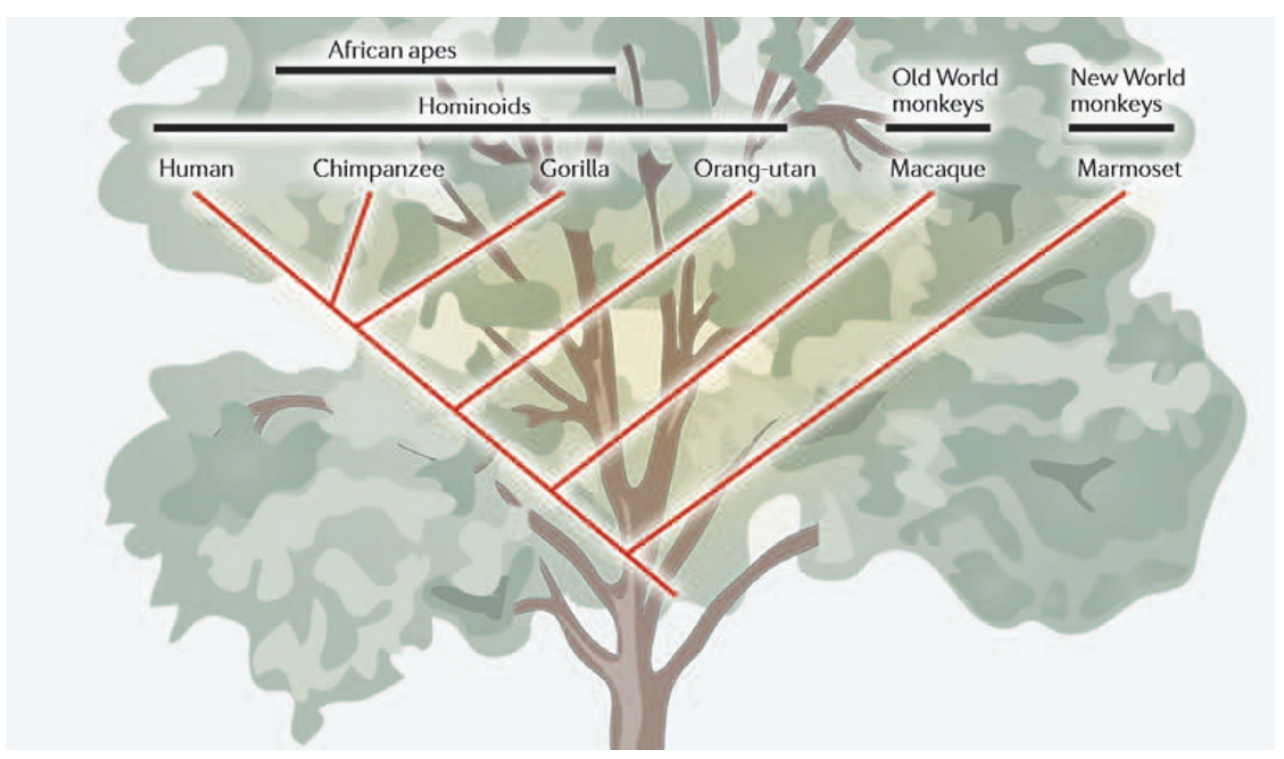

branches of the primate tree. The insights include branching order, relative divergence of groups within taxa, the placement of species for which phylogenetic relationships have been controversial, and a description of extensive variation in genome evolution rates. It is also possible to reconcile the taxonomy with suggested adaptive mechanisms; for example, in one family the tree confirms the existence of correlation between body size and evolutionary time. By contrast, some hypotheses are dispelled: the previously suggested 'hominoid slowdown', referring to a deceleration of evolutionary rate in human evolution, is in fact attributable to faster rates of evolution seen in the broader lineage, rather than in Homininae alone.

Although questions remain - for example, about the date and location of origin of primates - this new phylogeny provides a validated framework in which to interpret the evolution of human traits and our susceptibility to genetic or infectious disease, as well as genomic features such as the rates of recombination and rearrangement.

Tanita Casci

ORIGINAL RESEARCH PAPER Perelman, P. et al. A molecular phylogeny of living primates. PLoS Genet. 7, e1001342 (2011) FURTHER READING Philippe, H. et al. Resolving difficult phylogenetic questions: why more sequences are not enough. PLoS Biol. 9, e1000602 (2011)

Image is modified, with permission, from Samonte, R. V. \& Eichler, E. E. Nature Rev. Genet. 3, 65-72 (2002) (c) Macmillan Publishers Ltd. All rights reserved. 